\title{
Radio Spectrum Valuation by Applying the Artificial Neural Network Model
}

\author{
S. Malisuwan, N. Suriyakrai, and N. Madan
}

\begin{abstract}
As radio spectrum is a key resource for many technology-based industries and most promising industries of the future including broadcasting, telecommunications, military defense, industrial, medical and even domestic applications. These radio spectrum licenses are inputs into these business sectors that provide services to the public. The radio spectrum management and its valuation have turned out to be of vital importance in recent years due to the rapid development of mobile broadband communication. As a result of such popular demand of spectrum in this past decade, spectrum value is absolutely critical to policy analysis, as well as to encouraging investment in the telecom industry. This paper presents and proposes the radio spectrum valuation method by applying Artificial Neural Network model (ANN). The contributions in this paper could assist telecom policy makers to gain more understanding in development of radio spectrum valuation.
\end{abstract}

Index Terms-Radio spectrum, valuation, neural network, model.

\section{INTRODUCTION}

As penetration of mobile broadband and other services have experienced unprecedented demand, it has become extremely valuable national resource and therefore, its scarcity calls for efficient allocation more now than ever. There has been a rise of different spectrum theories advising on the nature of spectrum, most efficient usage and promotion of harmonization of spectrum. Most importantly an extension of spectrum topics is how spectrum should be regulated is the most significant aspect governments must take into consideration since managing spectrum resources has only began roughly half a century ago.

Due to the value that spectrum can provide, both industry and governments now view spectrum in terms of economic value that it can provide rather than just a resource necessary for some industries [1]. This is expected as over the past decade the world has seen the birth of technology and fast adoption of technological devices more than ever before and today the commercial mobile service industry has become a leading industry in the economy driving ICT and economic prosperity. Commercial mobile is not just an independent industry on its own but has become an enabler for opportunities for all sectors, creating a mobile ecosystem surpassing into all industry boundaries. This has resulted in policy makers, economists and regulators alike in

Manuscript received September 19, 2015; revised January 18, 2016. Financial support for this research paper is provided by National Broadcasting and Telecommunications Commission, Bangkok, Thailand.

The authors are with the National Broadcasting and Telecommunications Commission Bangkok, Thailand (e-mail: settapong.m@nbtc.go.th, nattakit.s@nbtc.go.th, navneet.m@nbtc.go.th). questioning, what should be the value of spectrum? How can we translate that value in financial terms? It is a simple question that has a complex answer that most policy makers and economists have yet to understand completely [2].

Spectrum valuation with econometric approach considers demand- and supply-side determinants of spectrum value. Supply-side determinants are, for example, license size, total spectrum to be auctioned, license length and set-aside. Demand-side determinants are GDP, GDP per capita, the number of subscribers, education level, ratio of revenue of telecommunication sector to GDP and long-run technological change [3]. In short, spectrum valuation with econometric approach is to find determinants of spectrum value while considering spectrum packaging, licensing procedure, payment schedule, domestic economy and market condition [4]. This approach involves five steps as follows.

- Model specification. Specify a model of linear or nonlinear relationship.

- Data collection. Collect historical data of all variables for use in estimation. Most data for

- Spectrum valuation is panel data which consists of time-series and cross-sectional data.

- Estimation. Estimate coefficients of the specified model. The estimation methods may be ordinary least squares, generalized least squares, two-stage least squares or others. The estimation techniques must be chosen properly to fix econometric problems.

- Evaluation of estimated model. Evaluate reliability of the estimated model and parameters with statistics such as t-statistics, F-statistics, R-squared and adjusted Rsquared.

- Forecasting. After evaluating the model, the last step is to forecast spectrum value given values of its determinants as in the estimated model.

Spectrum valuation by econometrics can be done in three main steps: (1) the first step is to apply econometric concept to develop a model to explain spectrum value in different countries. Such model is derived by finding important determinants of spectrum value and then constructing a regression equation to show the relationship between spectrum value and its determinants; (2) the second step utilizes statistical methods to estimate the model and; (3) the last step is to predict spectrum value of the studied country by replacing its values of determinants into the estimated equation [5].

Spectrum valuation model with artificial neural network is an econometric model classified as non-parametric or semi non-parametric model. This model is suitable for forecasting without knowledge of specific functional form between the left-hand and right-hand side variables or with 
complicated and non-linear functional form. Moreover, this model is resilient to change of data and data complexity. The Artificial Neural Network (ANN) is widely used in forecasting volatile processes such as optical character recognition, chemical procedure control, weather forecast, stock forecast and asset price forecast [6].

This paper aims to present and propose the radio spectrum valuation by applying the Artificial Neural Network model (ANN). This paper organizes as follows. Section II explains the concept of non-parametric estimation and ANN estimation. Section III presents the radio spectrum valuation by applying ANN. Section IV gives discussions and recommendations. The last section provides the conclusion of the paper.

\section{CONCEPT OF NON-PARAMETRIC ESTIMATION AND ARTIFICIAL NEURAL NETWORK ESTIMATION}

Classical econometrics favors parametric estimation since it is easy to compute and interpret, especially before the advent of computer with high processing power. A drawback of parametric estimation is its assumption about the linear functional form. Specifically, the ordinary least squares which is widely used in economics assumes that $y$ is $a$ linear function of $\mathrm{x}$ such that

$$
y=a+b x+e
$$

where $a$ and $b$ are coefficients to be estimated and $e$ is an error.

However, it admits that many economic relationships are complex and non-linear. Fig. 1 shows the relationship between $y$ and $x$ which is apparently linear but it is actually not. The actual relationship is $y=(x-1)(x-5)(x-7)(x-9)$ which is non-linear. In addition to the problem of non-linear relationship, another issue with the parametric estimation is the functional form is unknown.

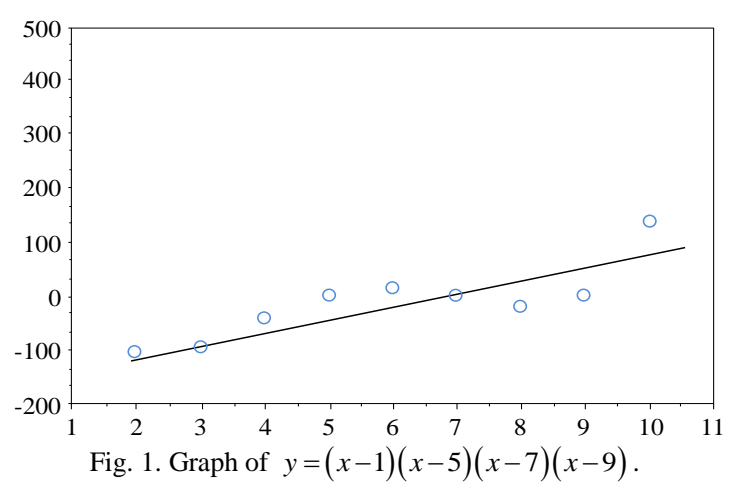

To avoid such problem of parametric estimation, nonparametric estimations such as artificial neural network estimation has been developed. Advantages of nonparametric estimation are that there is no need to specify a functional form and those non-linear and complex relationships can be estimated. The non-parametric estimation is suitable for a large sample. Fig. 2 and Table I compare parametric and non-parametric estimations as well as their pros and cons.

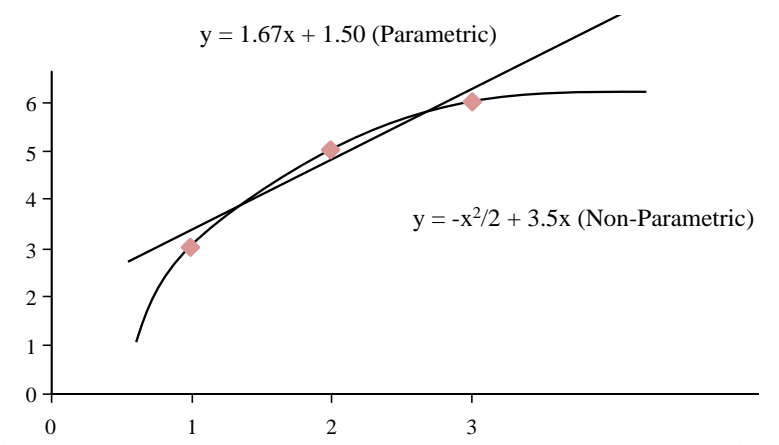

Fig. 2. Comparison between parametric and non-parametric estimations

TABLE I: PROS AND CONS OF PARAMETRIC AND NON-PARAMETRIC ESTIMATIONS

\begin{tabular}{|c|c|c|}
\hline \multicolumn{3}{|c|}{ ESTIMA } \\
\hline & Parametric method & Non-parametric method \\
\hline $\begin{array}{c}\text { Popular } \\
\text { technique }\end{array}$ & Least squares & Artificial neural network \\
\hline Pros & $\begin{array}{l}\text { - Simple and easy to interpret } \\
\text { - Applicable to small sample } \\
\text { - Suitable for linear relationship }\end{array}$ & $\begin{array}{l}\text { - Suitable for complex } \\
\text { relationship or relationship } \\
\text { with unknown functional } \\
\text { form }\end{array}$ \\
\hline Cons & $\begin{array}{l}\text { - Unsuitable for complex } \\
\text { relationship }\end{array}$ & - Large sample required \\
\hline
\end{tabular}

\section{A. Non-Parametric Estimation}

The principle of parametric estimation is to choose parameters to minimize the sum of prediction errors. For example, least squares estimation is to choose coefficients to minimize the sum of squared errors. However, in nonparametric estimation, since more complex functional forms are allowed, choosing a function that merely yields minimum prediction errors leads to overfitting and as a result the model cannot be used to fit or forecast other dataset. Fig. 3 shows an overfitted model. In Fig. 3, the actual relationship between $y$ and $x$ is linear but the estimated relationship swings up and down.

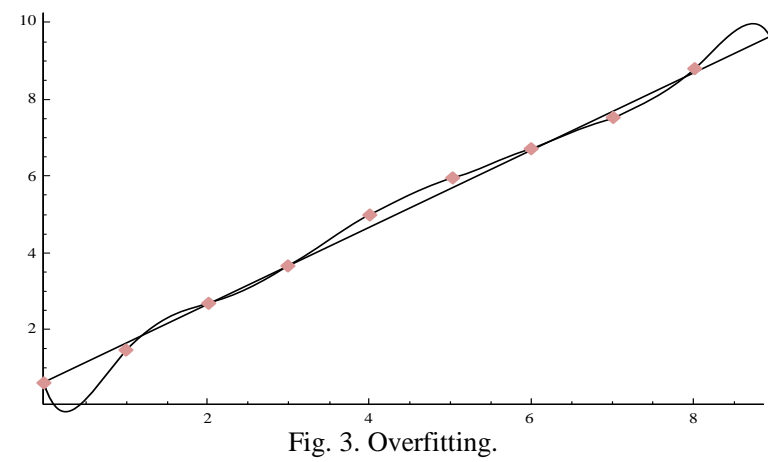

To resolve the overfitting problem, a non-parametric estimation will split the data into two datasets. The first data is used to construct a model and the second data set is used to test the model. The estimated errors in the first and second datasets must be insignificantly different. If the error in the first dataset is smaller than the second dataset, the model may have the overfitting problem.

\section{B. Artificial Neural Network Estimation}

The artificial neural network consists of three layers: input layer, hidden layer and output layer. The basic units of each layer are neuron nodes which receive information from input nodes, process the information and send outcomes to output nodes. Processing is done in two steps. In the first 
step, information from each input node are weighted and summed up. Then, the outcome is used in decision making by activation function as shown in Fig. 4. This function may be either linear or non-linear. Finally, the processed output from activation function is weighted again before sent to output node.

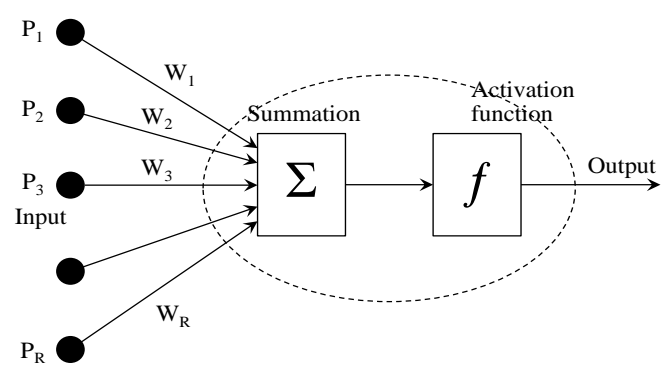

Fig. 4. Artificial neural network.

Widely used activation function is an S-shape curve such as Log-sigmoid (Fig. 5) whose range is between 0 and 1 as shown in the following equation.

$$
f(x)=\frac{1}{1+e^{-x}}
$$

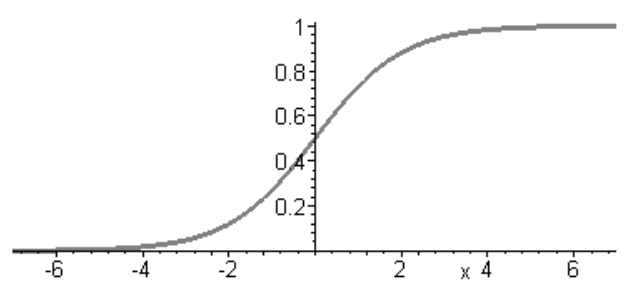

Fig. 5. Log-sigmoid function.

In addition to log-sigmoid function, the other popular functional form is hyperbolic tan sigmoid (Fig. 6) as shown in the following equation.

$$
f(x)=\frac{e^{x}-e^{-x}}{e^{x}+e^{-x}}
$$

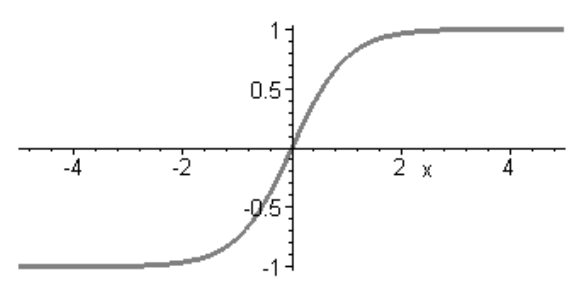

Fig. 6. Tan-sigmoid function.

\section{Node and Complexity of Artificial Neural Network}

In artificial neural network estimation, not only input and output data need to be selected, but the number of nodes which represent the complexity of artificial neural network must be chosen as well. The relationship from the estimation is shown in the following equation.

$$
Y=w 1 \cdot f 1(x)+w 2 \cdot f 2(x)+w 3 \cdot f 3(x)+\cdots+w n \cdot f n(x)(4)
$$

where $w$ is a weight for each node, $f$ is an activation function and $n$ is the number of nodes.

Equation (5) shows an example of the equation estimated by artificial neural network with one node.

$$
Y=w 1 \cdot f 1(x 1, x 2)=\frac{e^{2 \cdot x 1+3 \cdot x 2}-1}{e^{2 \cdot x 1+3 \cdot x 2}+1}
$$

In (5), there is only one $f(\cdot)$ since we specify the number of nodes to one. Equation (6) shows an example of the equation estimated by artificial neural network with two nodes.

$$
\begin{aligned}
Y & =w 1 \cdot f 1(x 1, x 2)+w 2 \cdot f 2(x 1, x 2) \\
& =3 \cdot \frac{e^{2 \cdot x 1+3 \cdot x 2}-1}{e^{2 \cdot x 1+3 \cdot x 2}+1}+4 \cdot \frac{e^{x 1+x 2}-1}{e^{x 1+x 2}+1}
\end{aligned}
$$

where $w 1$ and $w 2$ are weights for node 1 and 2, respectively. Since we set the number of nodes equal to two, there are two $f$ on the right-hand side of $Y$.

The examples above exhibit complexity of function to be estimated by an artificial neural network and they also show that the complexity is increasing in the number of inputs and nodes of the artificial neural network.

\section{Training Artificial Neural Network}

A popular training method is backward propagation which involves propagating input via outward neurons and then calculating an outcome for the next layer. This calculation is repeated layer-by-layer until the output layer is reached. If the calculation result has an error, the backward propagation step begins by updating weights between layers using errors between the target output and the output from the artificial neural network model. This error is propagated back into the network to update weights until the model yields minimum error. Such training method is called supervised training.

Each epoch of the typical backward propagation training is slow. The training method has been improved by Levenberg-Marquardt algorithm which is a method for numerical optimization. With this method, the parameter converges to the minimum in a short time. This method has been commonly used to train an artificial neural network.

To construct or train an artificial neural network, data are randomly split into three sets: (1) train set, (2) validation set and (3) test set. During the training, weights are adjusted to lower prediction errors in train set. The weight adjustment stops when the error in validation set starts to increase. Such termination helps prevent the overfitting problem. The test set is used to verify the final model before forecasting. This is also used to compare models.

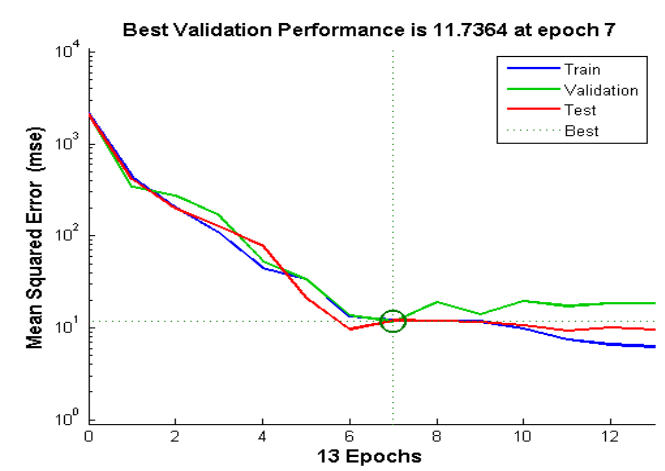

Fig. 7. Squared errors in train set, validation set and test set 
Fig. 7 shows mean squared errors of each epoch. According to the graphs, the adjustment stops at epoch 7 when the error in the validation set starts to rise.

\section{Forecasting SPECTRUM VAlUE USING BASIC VARIABLES}

First step is to forecast spectrum value using main variables to represent of demand and supply. These variables include GDP per capita, time trend and population density. GDP per capita is a determinant of purchasing power and a proxy of demand. Time trend reflects changes in consumer preferences on data service. Moreover, time trend is a proxy of technological change and network cost. Density is a determinant of supply which affects buildout cost [7].

\section{A. Forecasting with GDP per Capita}

It is conventional to convert spectrum value into a unit of price/MHz/pop. The most important demand-side determinant of spectrum value is purchasing power indicated by GDP per capita. Therefore, GDP per capita is used to forecast price/MHz/pop with an artificial neural network.

Basically, the spectrum price increases if GDP per capita rises. Moreover, the price elasticity of spectrum price is less than one or, in other words, the price increases proportionally less than the rise in GDP per capita.

\section{B. Forecasting with Time Trend}

Other than population income, another important determinant of spectrum price is time trend. It is very important to study the relationship between spectrum price and time trend and then use time trend to forecast spectrum value. The spectrum value can use both incomes per capital and time trend of forecast spectrum price.

\section{Forecasting with Population Density}

Previously, the paper presents the spectrum price with demand-side variables including population income and time trend. However, population density per kilometer which is an important determinant of buildout cost of mobile telecommunication network and supply. In theory, when population density increases, the service area becomes effectively smaller and thus revenue and spectrum value increase.

\section{Forecasting Spectrum Price Using Multi-variable Model}

The previous section forecasts spectrum price with basic variables that affects spectrum demand and supply. In this section, the basic variables from previous section which represent demand and supply factors such as income per capita, time trend and population density. In addition to these variables, we include variables that describe spectrum specific characteristics including license period, amount of paired spectrum.

\section{DISCUSSIONS AND RECOMMENDATIONS}

ANN is non-parametric model while most of statistical methods are parametric model that need higher background of statistic. Mathematical models are typically used to model a system when the system is not so complicated, but when the complexity of a system is increased the ANN can be applied for modeling.

Although the radio spectrum valuation by applying the ANN is a feasible approach, it has distinct advantages and disadvantages as shown in Table II.

TABLE II: PROS AND CONS OF PARAMETRIC AND NON-PARAMETRIC

\begin{tabular}{|c|c|}
\hline \multicolumn{2}{|c|}{ ESTIMATIONS } \\
\hline Advantages & Disadvantages \\
\hline $\begin{array}{l}\text { - Suitable when dependent and } \\
\text { independent variables have } \\
\text { complex relationships } \\
\text { - Large sample size increases } \\
\text { accuracy }\end{array}$ & $\begin{array}{l}\text { - Smaller sample size diminishes } \\
\text { the effectiveness of estimation } \\
\text { and prediction }\end{array}$ \\
\hline
\end{tabular}

To set a reserve price, the government policy is obligated to take all domestic stakeholders into consideration. Stakeholders are categorized into three groups. (1) Government: Government should receive reasonable revenue from economic rent that operators gain from acquired spectrum. (2) Consumers: Consumers should be able to receive quality mobile service enhanced by new spectrum at a fair price which can be achieved by competitive auction and mobile market. (3) Mobile operators: the reserve price should be set at the level that allows operators to make reasonable profit and have sufficient incentive to continuously invest in new technologies that benefit telecommunication services in the long run.

In summary, the reserve price should be set at a proper level, not too high or too low. A low reserve price coupled with limited number of bidders can lead to collusion which adversely affects the government revenue and consumer benefits. On the other hand, a high reserve price can discourage bidders and thus lead to an unsuccessful auction. Consequently, this creates obstruction to fully leverage the new spectrum as well as diminish the efficiency of resources management and the economic welfare of the nation.

\section{CONCLUSION}

Radio Spectrum is a scarce resource and it is very important to every country to understand its economic value to managing it efficiently. Estimating the value of radio spectrum faces with challenges because it needs to balance between economic value and social benefits. This paper presented the study of radio spectrum valuation by applying the Artificial Neural Network model. The paper analyzed and provided technical procedures to evaluate the value of it. The paper also recommended how to set a reserve price of the radio spectrum to maximize the efficiency of resources management and the economic welfare of the nation. This study contributes to governments and telecom regulators as a guideline to gain more understanding of the spectrum valuation.

\section{REFERENCES}

[1] P. Marks, K. Pearson, B. Williamson, P. Hansell, and J. Burns, "Estimating the commercial trading value of spectrum," A Ofcom Report by Plum Consulting, pp. 20-33, July 2009. 
[2] J. Alden, "Exploring the value and economic valuation of spectrum," Geneva: International Telecommunication Union (ITU), pp. 5-20, April 2012.

[3] P. Cramton, Spectrum Auctions, February 2001, pp. 23-35.

[4] G. Madden, I. Sağlam, and A. Morey, "Auction design and the success of national 3G spectrum auctions," TÜSIAD-Koç University Economic Research Forum Working Paper Series, pp. 5-9, February 2010.

[5] J. J. Heckman and E. Leamer, Handbook of Econometrics, Elsevier, December 2007, vol. 6, part B, pp. 4779-5143.

[6] K. Gurney, An Introduction to Neural Networks, CRC Press, 1997, p. 234.

[7] Plum Consulting, "Methodologies for valuing spectrum: Review of the experts' report," A Report for Vodafone, pp. 5-9, March 2011.

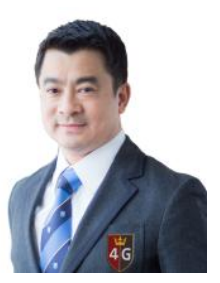

S. Malisuwan was born on March 24, 1966 in Bangkok, Thailand. He was awarded the full scholarship from Thai government for $\mathrm{PhD}$ in electrical engineering (telecommunications), specializing in mobile communication systems from Florida Atlantic University (State University System of Florida), Boca Raton in 2000. He received his MSc degree in electrical engineering in mobile communications system from George Washington University in 1996 and was awarded the First Class Honors, Gold Meda Award and Outstanding Cadet Award by the university. He also achieved the MSc degree in electrical engineering in telecommunication engineering from Georgia Institute of Technology in 1992. Furthermore, he achieved military education from the Special Warfare Center, Thailand, specializing in ranger and airborne courses in 1989 and 1988 respectively. He is currently the vice chairman and board commissioner of the National Broadcasting and Telecommunications Regulator in Bangkok, Thailand. He was awarded the "Science Towards the Excellence in 2013" by The Senate Standing Committee on Science, Technology, Communications and Telecommunications. His research interests are in electromagnetic, efficient spectrum management and telecommunications policy and management.

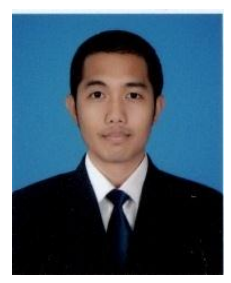

N. Suriyakrai was born in Khonkhaen, Thailand on March 22, 1987. He received his bachelor degree of liberal arts in Japanese language from Thammasat University in 2010. He has been working as an assistant to vice chairman in the National Broadcasting and Telecommunications, Bangkok, Thailand since November 2012. His research interests are in technology management and spectrum management.

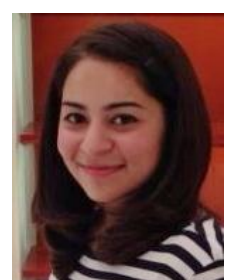

N. Madan was born in Bangkok, Thailand on April 22, 1987. She received her bachelor degree of business administration in international business management from Mahidol University in 2008, and received the master of science degree in strategic management and marketing, Middlesex University, London, United Kingdom. She has been working as an assistant to vice chairman in National Broadcasting and Telecommunications, Bangkok, Thailand since January 2012. Her research interests are in spectrum management strategic flexibility, market orientation and environmental uncertainty in fast clock speed industries. 